\title{
Implementación de procesos de control de calidad en la actualización de series cartográficas urbanas mediante combinación de CAD y SIC
}

\author{
Implementation of quality control processes in the updating \\ of urban cartographic series through a \\ combination of CAD and CIS
}

\author{
Eulàlia Torrent Foz' \\ Ángel Luis Muñoz-Nieto² \\ Diego González-Aguilera ${ }^{3}$ \\ Pablo Rodríguez-Conzálvez ${ }^{4}$
}

Recibido 25 de octubre de 2020; aceptado 25 de marzo de 2021

\section{RESUMEN}

El presente artículo presenta un conjunto de desarrollos y herramientas diseñadas con el fin de automatizar el mayor número posible de los procesos de control de calidad que se realizan sobre una serie cartográfica durante las fases de mantenimiento y actualización dentro de un contexto municipal. Dado que los tiempos y recursos empleados en estas tareas son considerables, se ha diseñado una metodología que aprovecha las posibilidades de los entornos CAD y SIG para agilizar y optimizar la detección de las discrepancias entre la representación cartográfica y los parámetros establecidos por el organismo cartográfico competente, facilitando así la localización de estos errores y su

Ayuntamiento de Sabadell, España, correo electrónico: eulalia.tf.et@gmail.com.

ORCID: https://orcid.org/0000-0002-5422-2085

Universidad de Salamanca, España, correo electrónico: almuni@usal.es

ORCID: https://orcid.org/0000-0002-0814-1639

Universidad de Salamanca, España, correo electrónico: daguilera@usal.es.

ORCID: https://orcid.org/0000-0002-8949-4216

Universidad de León, España, correo electrónico: p.rodriguez@unileon.es

ORCID: https://orcid.org/0000-0002-2657-813X 
posterior revisión y corrección. El proceso de automatización se ejemplifica con las series cartográficas a gran escala del Ayuntamiento de Sabadell, lográndose una importante minimización de la intervención de los técnicos.

Palabras clave: cartografía, control de calidad, Sistema de Información Geográfica, actualización cartográfica, detección automática de errores.

\section{Abstract}

This article presents a set of developments and tools designed to automate the greatest possible number of quality control processes carried out on a cartographic series during the maintenance and updating phases, within a municipal context. Given that the time and resources employed in these tasks are considerable, a methodology has been designed that takes advantage of the possibilities of the CAD and GIS environments to speed up and optimise the detection of discrepancies between the cartographic representation and the parameters established by the competent cartographic body, thus facilitating the location of these errors and their subsequent revision and correction. The automation process is exemplified by the large scale cartographic series of Sabadell City Council, achieving a significant minimization of the intervention of technician.

Key words: cartography, quality control, Geographic Information System, cartographic update, automatic error detection.

\section{Introducción}

Los trabajos de mantenimiento y actualización de una base cartográfica dentro de una organización suponen uno de los procesos más importantes de las tareas de la misma, consumiendo la mayor parte de los recursos de la sección o departamento (Ariza \& Rodríguez, 2018). A pesar de su importancia, los procesos de control de calidad de la cartografía suelen quedar en segundo plano, debido a la falta de medios, de recursos humanos y/o económicos. Pero con el tiempo se hace evidente la necesidad de disponer de un sistema de gestión para garantizar la calidad de la cartografía (Gallego-Priego, 2017). Esto implica documentar procesos, procedimientos y metodologías para garantizar la calidad del producto respecto a unos requisitos establecidos (Ariza-López, 2013).

El presente artículo busca contribuir a la modernización y mejora del proceso de control de calidad de la cartografía de los ayuntamientos, de modo que se supere la revisión manual y las tareas repetitivas que se realizan sobre las hojas de una serie cartográfica. Para ello se presenta un conjunto de herramientas que permiten automatizar un gran número de tareas dentro del procedimiento de control de calidad utilizando, en la medida de lo posible, software libre y de código abierto. Con el objeto de ilustrar la metodología y herramientas implementadas, se ejemplificarán los resultados con el caso de la cartografía a gran escala del ayuntamiento de Sabadell. En todo caso, las 
herramientas desarrolladas están abiertas a ser modificadas para ajustarse a las necesidades y casuísticas concretas de otra organización (corporaciones municipales principalmente) según sus necesidades específicas o a ampliar su rango de uso incorporando nuevos parámetros de control para depurar todavía más el producto cartográfico final.

\subsection{Contexto de trabajo}

En la comunidad autónoma de Cataluña, para que la cartografía generada por una administración local tenga carácter oficial y pueda integrarse en el Registro Cartográfico de Cataluña (RCC), es necesario que haya sido elaborada según las normas y estándares establecidos por la Comisión de Coordinación Cartográfica de Cataluña (CCCC). Así se establece en el Plan Cartográfico de Cataluña que indica que la cartografía registrada debe tener un buen nivel de actualización y debe estar disponible según las normas y estándares mínimos de calidad (DOGC, 2010). De este modo, cada vez que se realiza una solicitud para registrar una nueva versión de un conjunto cartográfico al RCC, el Instituto Cartográfico y Geológico de Cataluña (ICGC) realiza un control de calidad sobre la totalidad de la documentación presentada para evaluar si esta puede registrarse o si presenta deficiencias.

En el contexto específico del Ayuntamiento de Sabadell se han realizado distintos trabajos para adaptar la cartografía municipal a los estándares oficiales documentándose estos procesos de creación y mantenimiento de la cartografía municipal mediante el Pliego de especificaciones técnicas de la cartografía topográfica 2D del ayuntamiento de Sabadell a escalas 1:500 y 1:1 000, basado en el estándar elaborado por la Comisión de Coordinación Cartográfica de Cataluña (CCCC, 2016).

Las incidencias registradas en los procesos cartográficos, relacionadas con la calidad de representación, evidenciaron la necesidad de disponer de herramientas de control interno para evitar este tipo de problemas en el futuro. El compromiso de mantener la cartografía actualizada y registrada para su consulta de acuerdo con los estándares establecidos, obliga a la realización de entregas periódicas al RCC y esto requiere la realización de controles de calidad con mucha frecuencia para que la cartografía esté convenientemente actualizada, y por tanto refleje con prontitud los cambios que se producen en el territorio. En consonancia con la norma internacional ISO 19157-1 se detallan procedimientos de búsqueda y corrección de errores de consistencia lógica y exactitud temática.

Los procesos de actualización cartográfica y control de calidad llevados a cabo manualmente llevan implícitos una serie de errores sistemáticos y accidentales vinculados a la dinámica de trabajo del operador como su pericia, concentración, agudeza visual, etc. Por ello es aconsejable la implementación de una metodología de trabajo sistemática y unas herramientas que permitan automatizar el proceso de control de calidad mejorando los resultados en términos de reducción de costes de tiempo y fiabilidad de los resultados. 


\subsection{Objetivos}

El objetivo principal de las herramientas desarrolladas es automatizar el proceso de control de calidad que se lleva a cabo sobre una serie cartográfica evitando la revisión manual y repetitiva de las hojas cartográficas que componen la serie para verificar que cumplen con los estándares mínimos de calidad y contenido. Como estándares de referencia se han establecido los recogidos según el Pliego de especificaciones técnicas de la cartografía topográfica 2D del ayuntamiento de Sabadell a escalas 1:500 y 1:1 000 en cuanto a la representación de sus elementos. Como resultado final del control de calidad se identificarán, en las hojas cartográficas, los elementos que no cumplen con las características definidas en el catálogo de representación de elementos de la serie, localizando y clasificando los errores detectados para poder corregirlos más fácilmente.

Para comprobar la conformidad de la cartografía municipal con las especificaciones, se empleará el diccionario o catálogo de representación de elementos de la serie cartográfica asociado al control de calidad. El catálogo es un documento en forma de tabla en la que se relacionan los distintos elementos que pueden contener las hojas de la serie cartográfica y se definen, de forma unívoca, las características que deben cumplir estos elementos.

Seguidamente se desglosa el objetivo principal del trabajo en los siguientes objetivos específicos:

- Proponer una metodología para la automatización en la detección y corrección de errores cartográficos.

- Diseñar e implementar un set de herramientas flexible, que permita añadir nuevos inputs (nuevos tipos de error a detectar).

- Elaborar automáticamente informes de los errores detectados en las hojas procesadas.

- Crear marcadores de errores para facilitar su localización en el entorno CAD.

- Aplicar la metodología propuesta a un supuesto práctico.

- Validar los resultados del ensayo.

\section{Materiales y métodos}

Para describir a fondo los procesos de control de calidad y las metodologías de trabajo, se presentarán inicialmente los materiales empleados, para centrarse posteriormente en los aspectos metodológicos y de desarrollo implementados.

\subsection{Materiales}

Las herramientas software empleadas para los procesos de automatización se han desarrollado en dos entornos que se denominaran "entorno CAD" y "entorno SIG". El "entorno CAD" constituye un entorno gráfico donde se puede ver, editar y manipular los ficheros gráficos de la serie cartográfica (en formato DGN). Para ello se empleará el software MicroStation v8i (Bentley, 2020) y el módulo de Visual Basic integrado para desarrollar funciones o rutinas personalizadas. El objetivo principal será exportar toda la información de los elementos presentes 
en los ficheros gráficos a un fichero de texto estructurado, que se empleará como input del siguiente proceso que se ejecutará en entorno SIG.

El "entorno SIG" contempla el empleo de herramientas propias de un SIG para llevar a cabo las tareas relacionadas propiamente con el control de calidad. Nos referimos a tareas como cruzar información o analizar los datos obtenidos de cada hoja tras su comparación con el modelo de datos y la simbología asociada, y de este modo detectar los errores de representación. Para ello se escogió trabajar con el software libre QGIS (QGIS, 2020) y el lenguaje de programación Python.

\subsection{Metodología}

Para sintetizar como se van a estructurar, ordenar y relacionar las distintas fases y tareas que conforman el protocolo de automatización del procedimiento de control de calidad se presenta el diagrama mostrado en la Figura 1.

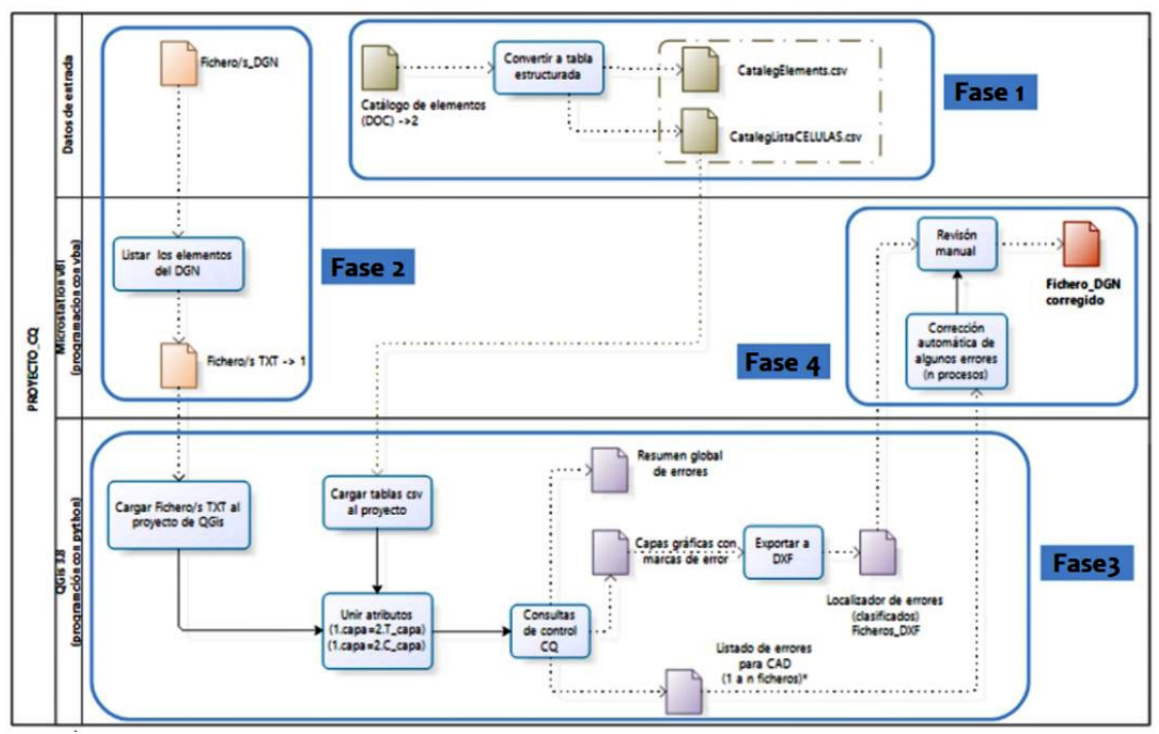

Figura 1. Diagrama conceptual del procedimiento de control de calidad.

Sobre dicho diagrama se han realizado cuatro agrupaciones (fases) que servirán en adelante para identificar inequívocamente la parte del proyecto que se está describiendo en cada momento. Dichas fases son:

- Fase 1. Preparación de datos de control

Su propósito es estructurar la información obtenida de los ficheros de especificaciones que acompañan la serie cartográfica y que servirán para establecer las normas de representación que deben cumplir los elementos de la cartografía. 
- Fase 2. Conversión DGN a TXT

El objetivo de esta fase es la extracción de la información de las hojas cartográficas de modo que pueda ser incorporarla al SIG, en el que posteriormente se implementarán los algoritmos para la detección de errores y discrepancias.

Aunque QGIS permite importar ficheros CAD, esta importación es costosa en tiempo y peso porque incorpora la geometría de todos los elementos. Y, en este caso, no nos interesa tanto la geometría de los objetos como los atributos de representación de los mismos. Por este motivo se optó por trabajar con un fichero de texto que contuviera la información de representación de los objetos del fichero grafico geolocalizada como si fuera un objeto puntual (tomando como localización el punto inicial en el caso de elementos lineales y el centroide para los elementos poligonales). El fichero de texto es fácil de incorporar al SIG y muy ligero y fácil de analizar.

- Fase 3. Control de calidad-Detección de errores

Se trata en esta fase de diseñar un proceso de detección e identificación de errores en el SIG. Para ello se definirán varias rutinas.

- Fase 4. Corrección de errores

Esta última fase se propone indicar al operador, de forma explícita, cómo se resolverían los distintos tipos de errores.

En un proceso de control de calidad es necesario que previamente se hayan definido los tipos de errores que se están buscando, explicitando las diferencias entre las características previstas de un elemento cartográfico analizado y las características obtenidas. En el presente trabajo se empleó el modelo de datos y la simbología del catálogo de representación de elementos de la serie para definir los errores que se van a buscar. Si bien es recomendable tener en cuenta la experiencia previa para determinar la tipología de errores esperados.

A continuación, se listan los tipos de errores a localizar agrupándolos en primera instancia en función del análisis interno por niveles (capas):

- Representación de elementos:

- Niveles con elementos que no se representan según sus atributos de color, tipo de línea o grosor.

- Objetos orientados que, por definición, deberían presentar un valor de orientación igual a cero.

- Consistencia de datos:

- Niveles con elementos que no corresponden al tipo esperado por el propio nivel. Por ejemplo, niveles que esperan elementos lineales, pero contienen elementos de tipo texto, punto, formas, etc.

- Depuraciones de información. Por ejemplo, en el caso de elementos lineales, detección y eliminación de elementos con longitud cero.

- Valores de texto y formato:

- Revisión del formato de algunos textos. Por ejemplo, en el caso de las anotaciones de cotas altimétricas identificar si hay algún elemento con 
un separador decimal distinto al definido en el modelo de datos, el número de decimales que contiene, etc.

- Detección del contenido del texto corresponde con el esperado para el nivel. Por ejemplo, que la anotación de un elemento concreto como una rampa sea "Rampa" en lugar del esperado "rampa".

Más concretamente, en el presente trabajo se ha automatizado la detección de los siguientes once tipos de errores:

- Error 1: Nivel lineal con elementos que no son líneas o polilíneas.

- Error 2: Nivel puntual con otros tipos de elementos.

- Error 3: Nivel de texto con otros tipos de elementos.

- Error 4: Elementos lineales de longitud cero.

- Error 5: Elementos con color diferente al asignado al nivel ( $\neq-1)$.

- Error 6: Elementos con tipo de línea diferente al asignado al nivel ( $\neq$ ByLevel).

- Error 7: Elementos que con grosor diferente al asignado al nivel ( $\neq-1)$.

- Error 8: Elementos puntuales no orientables con valor angular distinto a cero.

- Error 9: Nivel de texto (valor de cotas altimétricas) con separador decimal "." en lugar del esperado ",".

- Error 10: Nivel T-cota_corba_nivell con separador decimal "." en lugar del esperado ",".

- $\quad$ Error 11: Clase diferente de 0 (= "primaria").

Este listado de errores no pretende ser exhaustivo. Por ello, las herramientas diseñadas son escalables y permitirían definir otras casuísticas de errores como, detectar la presencia de algún elemento en un nivel que no aparece en el catálogo; elementos puntuales representados con definiciones de células fuera de las esperadas; representaciones de texto no conformes con el formato definido... Cuantos más tipos de errores se puedan considerar, más depurada podrá estar la cartografía y por ende aumentará su calidad.

\subsubsection{Preparación de datos de control (fase 1)}

El catálogo de representación de elementos es una tabla que complementa los mapas de una serie cartográfica indicando los atributos gráficos que debe tener cada elemento para su correcta representación. Dicha información será empleada para asegurar que las hojas cartográficas analizadas se ajustan a las especificaciones de la serie. En la Figura 2 se muestra el catálogo de representación de elementos que recoge tanto el modelo de datos como la simbología.

El primer paso es manipular la tabla del catálogo de representación de elementos para adecuar los nombres de campos y datos de la tabla y poder trabajar con la información que contiene. Como formato preferente se escogerá el CSV, y se generarán dos ficheros: 
- Catálogo de representación de elementos propiamente dicho y que contendrá el listado completo del catálogo con los campos de información renombrados para evitar confusiones.

- Catálogo de células que contendrá la clasificación de los tipos de objetos puntuales que puede contener cada nivel con una relación 1 a 1. Las células son un conjunto de líneas o elementos que se agrupan para ser tratadas como un único objeto puntual. Se usan para representar elementos concretos como por ejemplo un árbol, un banco, una farola o una tapa de registro. De modo que, a partir de una definición de célula, se puede representar fácilmente tantas veces como sea necesario ese objeto.

\begin{tabular}{|c|c|c|c|c|c|c|c|c|c|c|c|c|c|c|c|}
\hline \multicolumn{16}{|c|}{ CATALEG D'ELEMENTS. Cartografia topogràfica 2D } \\
\hline \multicolumn{13}{|c|}{ Hidrografia-Obres hidràuliques } & \multicolumn{3}{|l|}{$\begin{array}{l}\text { CARTOCPAFA } \\
\text { TEMARTA }\end{array}$} \\
\hline Bement & Fitxa & Color & Estil & Gruind & Bement gràific & Font & Just & Alg & Amp & Nom cerla & Escala & Angle & $\begin{array}{c}\text { Dades } \\
\text { externes }\end{array}$ & Ethaustivita & Representacid \\
\hline LLinib de costa & HD_01 & ss & 0 & 0 & Line Steing-Line & & & & & & & & & No & 100 \\
\hline \begin{tabular}{|l|l|} 
LRivi aigues permenerts \\
\end{tabular} & HD_.02 & 105 & 0 & 0 & Line String-Line & & & & & & & & & $s$ & si \\
\hline 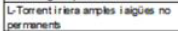 & HD_es & 105 & 2 & $\circ$ & Une String-Line & & & & & & & & & $\mathbf{s}$ & s \\
\hline 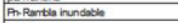 & HD_.04 & 105 & 1 & 0 & Shape & & & & & & & & & NO & no \\
\hline \begin{tabular}{|l|l|} 
LAbl \\
\end{tabular} & HD_05 & 74 & 0 & 0 & Line Sting-Line & & & & & & & & & no & 10 \\
\hline \begin{tabular}{|l|l} 
LCanal otra \\
\end{tabular} & HD_ 00 & 7 & 0 & 0 & Line Sting-Line & & 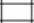 & 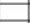 & & & & & & $s$ & $s$ \\
\hline LCanal de terta & HD_ 07 & 7 & 0 & 0 & Line Sting-Line & & & & & & & & & $s$ & s \\
\hline L-Siguia & $\mathrm{HD}-0$ & 98 & 0 & 0 & Line Sving-Line & & & & & & & & & $\mathrm{s}$ & si \\
\hline 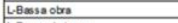 & HD, 09 & 98 & 0 & 0 & Line Sting-Line & & & & & & & & & $s$ & $s$ \\
\hline LExsa deterta & HD_ 10 & 98 & 0 & 0 & Line String-Line & & & & & & & & & $s$ & $s$ \\
\hline Lffoine & HD 11 & 98 & 0 & 0 & Line String-Line & & & & & & & & & $s$ & st \\
\hline A-Pation de pla die & H1O_11-01pol & 98 & 0 & 0 & Shape & & & & & & & & s & $s$ & st \\
\hline LFou & HD 12 & 0 & 0 & 0 & Line String-Line & & & & & & & & & $s$ & s \\
\hline LRekica de des puls & $\mathrm{HD}, 13$ & 102 & 0 & 0 & Line String-Line & & & & & & & & & s & st \\
\hline REntornal & HD_14 & 102 & 0 & 0 & Cetia & & & & & BABOFa & vasose & orent & & s & s \\
\hline RSinboldefort & HD_.15 & 98 & 0 & 0 & Coth & & & & & Font, Foirt & vasas & orent & & s & s \\
\hline LFont & HD_16 & 98 & 0 & 0 & Line String-Line & & & & & & & & & s & s \\
\hline PHidrant & HD_17 & 102 & 0 & 0 & Coth & & & & & \begin{tabular}{|l|} 
ROH \\
\end{tabular} & 1.0 & 0 & & s & $s$ \\
\hline PBocadiotion & HD_-18 & 102 & 0 & 0 & Cet is & & & & & BREC, Brea & vatas & 0 & & $s$ & s \\
\hline RBoca incendis & HD_19 & 105 & 0 & 0 & Cet is & & & & & PAC, PBQ & vatesien & 0 & & $\mathrm{~s}$ & sis \\
\hline LRerd & HD 20 & 105 & 3 & 0 & Line String - Line & & & & & & & & & s & $s$ \\
\hline LTartentiritera estes & HD_21 & 105 & 2 & 0 & Line String - Line & & & & & & & & & s & sa \\
\hline \begin{tabular}{|l|l} 
LEk cansi dora \\
\end{tabular} & HD 22 & 7 & (Arrow) & 0 & Line String-Line & & & & & & & & & no & no \\
\hline \begin{tabular}{|l|} 
LEx canal de terrs \\
\end{tabular} & HD_.23 & 7 & (Atrow) & 0 & Line String-Une & & & & & & & & & No & No \\
\hline \begin{tabular}{|l} 
L-Rejca de canal de des guls \\
\end{tabular} & HD_24 & 102 & 0 & 0 & Line String-Line & & & & & & & & & No & NO \\
\hline LEK de riv irrend & $\mathrm{HO}, 25$ & 105 & 4 & 0 & Line String - Line & & & & & & & & st & $s$ & si \\
\hline LEK de brent inisa & $H D, 26$ & 105 & 3 & 0 & Line String - Line & & & & & & & & $\mathrm{s}$ & $s$ & $s$ \\
\hline
\end{tabular}

Figura 2. Muestra del catálogo de representación de elementos con los atributos de representación.

Fuente: Pliego de especificaciones técnicas de la cartografía topográfica 2D del Ayuntamiento de Sabadell a escalas 1:500 y 1:1 000.

En el primer fichero, se mantendrán todos los campos de la tabla, pero renombrados para evitar caracteres problemáticos y garantizar que se podrá importar correctamente cuando lo llamemos desde el entorno SIG. Después de renombrar los campos, se modificarán los valores del campo TypeElement, el cual recoge la descripción del tipo de elemento gráfico que espera el nivel (puntuales, lineales, poligonales y de texto).

La necesidad de creación del segundo fichero de células se debe al hecho que en el listado original hay niveles que admiten distintas representaciones de objetos puntuales de tipo célula. Para que esta relación 1: $\mathrm{N}$ no sea un problema al relacionarla con la información obtenida de los ficheros gráficos DGN, se crea esta tabla auxiliar de soporte. De modo que la nueva tabla indique todas 
las combinaciones de nivel/célula que puede admitir el fichero DGN según el catálogo.

\subsubsection{Conversión DGN a TXT (fase 2)}

Esta segunda fase se desarrolla en el entorno CAD (Microstation) donde se creará una rutina o macro para exportar toda la información de los elementos gráficos de un fichero DGN (Figura 3) y almacenarla en un fichero de texto estructurado (TXT), de modo que se puedan consultar los atributos de representación de los elementos y otras características propias al tipo de elemento. Esta información será imprescindible para que el control de calidad pueda detectar qué elementos están mal representados o si contienen alguno de los tipos de errores previstos.

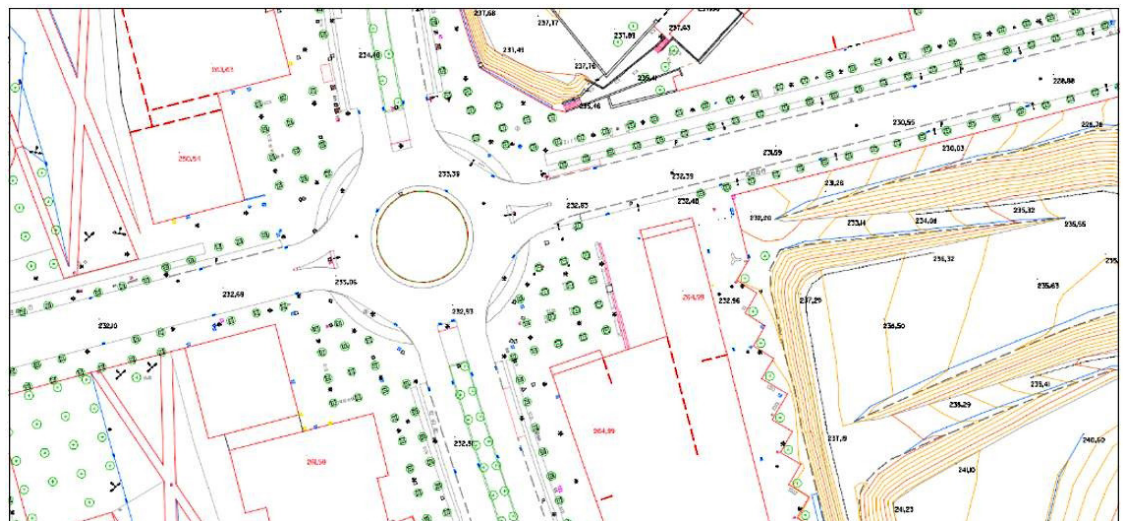

Figura 3. Muestra de la base cartográfica de Sabadell: recorte de la hoja 392-34-26.dgn. Fuente: Ayuntamiento de Sabadell.

La macro de MicroStation realiza un bucle de selección de los elementos contenidos en cada nivel y, en función del tipo de elemento, graba sus características específicas en el fichero de texto. Además, añade una cabecera al fichero de texto que contiene los nombres de los campos de información a exportar. En la Tabla 1 se recogen los tipos de elementos con los que se trabajará.

En la rutina se establece una operación del tipo Select-Case en función de los valores de TypeElement esperados. Esta instrucción es escalable, tal que podrá admitir otros casos para poder identificar y valorar si se trata de otros tipos de elementos gráficos a considerar que deben estar en la hoja cartográfica, si hay que eliminarlos o si se trata de elementos de definición del propio fichero de MicroStation que no afectan a la información gráfica. 
Tabla 1. Tipos de elementos cartográficos y su correspondencia en Microstation

\begin{tabular}{cc}
\hline Tipo de elemento cartográfico & $\begin{array}{c}\text { Tipo de elemento de } \\
\text { Microstation (TypeElement) }\end{array}$ \\
\hline Elemento puntual & 2 \\
\hline Elemento lineal & $4-3$ (menos frecuente: 15, 16) \\
\hline Elemento poligonal & 6 \\
\hline Elemento de texto & 17 \\
\hline
\end{tabular}

Como resultado de la exportación masiva del proceso por lotes, se obtendrán $n$ ficheros similares (uno por cada hoja cartográfica procesada) al ejemplificado en la Figura 4.

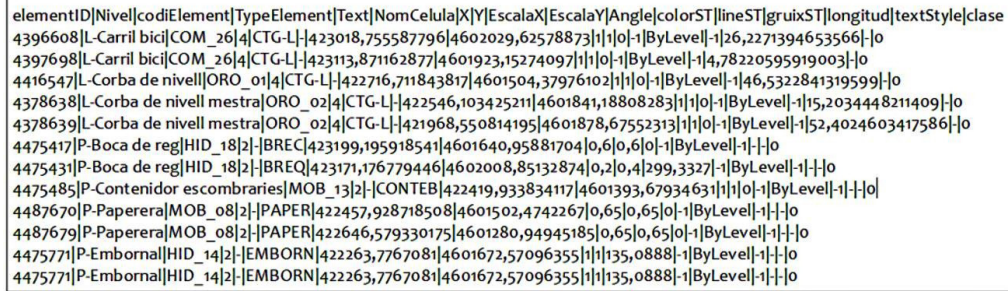

Figura 4. Ejemplo del fichero de texto obtenido al ejecutar la macro sobre un fichero DGN.

\subsubsection{Control de calidad (fase 3)}

En esta etapa es en la que se comprueba la exactitud de los elementos de cada fichero DGN (hoja de la serie cartográfica). En concreto se chequea la fidelidad de la clasificación y de los atributos esperados. Para ello se siguen los procesos que a continuación se detallan.

\section{Carga de datos iniciales en el entorno SIC}

Como datos iniciales se parte de los ficheros obtenidos al finalizar las fases 1 y 2 expuestas anteriormente. En el entorno de trabajo de QGIS se crea una

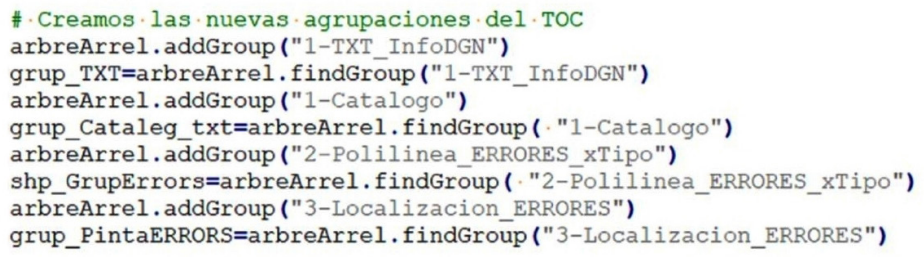

Figura 5. Código Python para la creación de las agrupaciones. 
estructura de agrupaciones que permita almacenar, de forma ordenada, tanto los datos de entrada, como los resultados que se obtendrán al ejecutar cada una de las fases que conforman el control de calidad (Figura 5).

\section{Enlace de información por unión de tablas}

Seguidamente se enlaza la información de los ficheros DGN con los datos del catálogo, mediante la indicación del campo común entre ellos. El resultado es una unión virtual de información que se añade a la tabla principal (receptora) como datos anexados.

Para evitar ambigüedades, la relación de las dos tablas del catálogo de representación de elementos con cada uno de los ficheros de datos se realiza mediante una correspondencia del tipo 1:1. El proceso de unión está automatizado mediante código Python. En la Figura 6 se muestra un modelo relacional con los campos que se van a utilizar para realizar la unión entre las tablas.

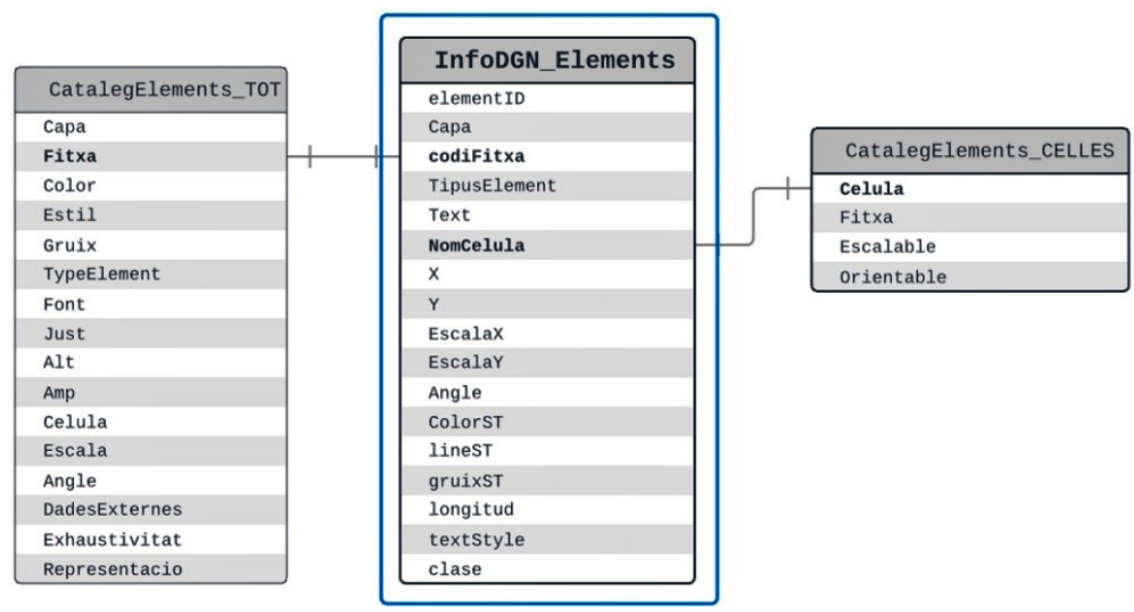

Figura 6. Unión de las tablas del catálogo con la tabla que contienen la información de los elementos de una hoja cartográfica concreta.

\section{Definición e identificación de errores}

En este proceso vamos a definir los errores que podemos localizar por diferencia entre el valor real del elemento y su valor esperado según el catálogo.

A continuación, se muestran las pautas seguidas para la automatización del proceso de detección de estos errores en el SIG y la pauta establecida para obtener resultados. Con ellas se persigue la obtención de:

- Un resumen global de errores detectados en todos los ficheros de datos procesados.

- Un listado con todos los errores detectados. 
- Marcadores de errores que podamos exportar para facilitar la corrección de las hojas cartográficas.

La rutina sigue una pauta interactiva: cada vez que se procesa un tipo de error y, para cada tipo de error, se establece una expresión o función. Esta expresión se usará, a modo de filtro, para detectar los elementos que cumplen con la condición establecida (Figura 7).

También se definen dos funciones adicionales, que se ejecutan cada vez que se busca un tipo de error. La primera sirve para obtener un listado o reporte de todos los errores detectados en el fichero. La segunda crea una línea que une todos los errores del mismo tipo detectados. Esta línea será de gran utilidad como guía y referencia en la fase final del proceso de revisión, limpieza y corrección de los errores sobre las hojas cartográficas.

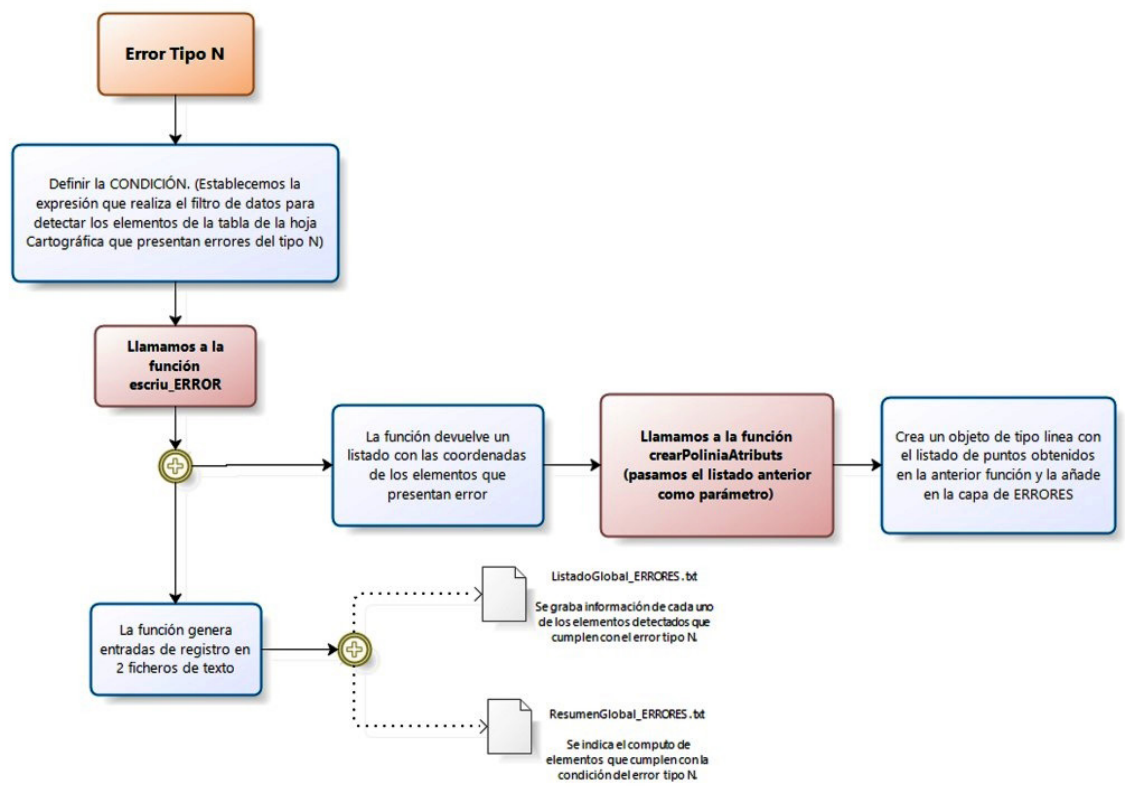

Figura 7. Esquema del proceso de detección de errores.

\section{Localización y representación de resultados}

En este proceso se genera una salida gráfica cuya misión es ayudar al técnico a localizar los errores detectados. Para ello se crean un conjunto de líneas que unen los puntos donde están localizados los errores de una determinada tipología, asignando a estos puntos información sobre las propiedades del error detectado.

De este modo, la rutina para el operador de control de calidad consiste en recorrer el circuito definido por las líneas de detección de errores tantas veces como tipos de errores hayan sido definidos. En la Figura 8 se recoge un diagrama 
conceptual del proceso y de la creación de la capa gráfica de errores. Como se aprecia, cada vez que se procesa un tipo de error, el circuito devuelve una línea que se incorpora a la capa de errores.

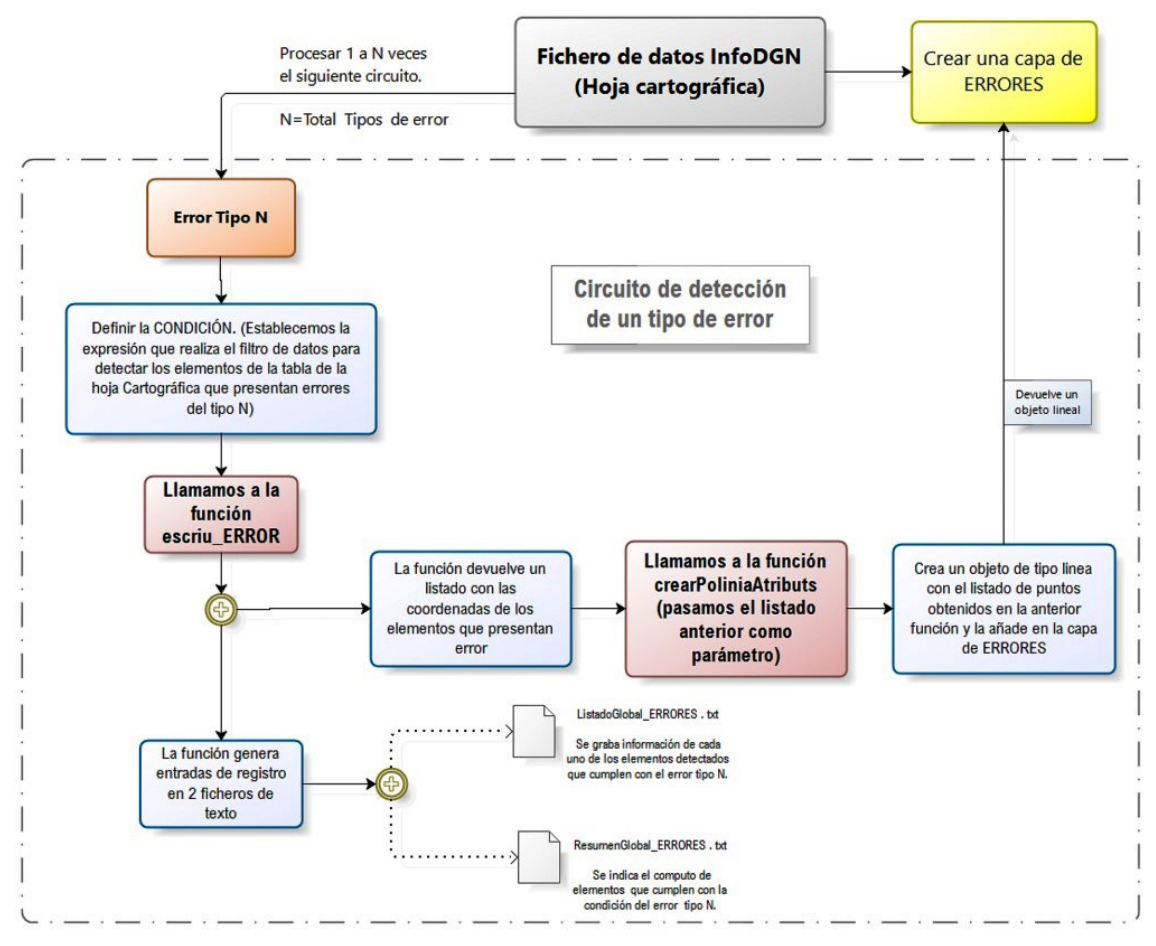

Figura 8. Esquema de creación de la capa de errores.

Como ya se indicó el presente trabajo parte de la definición de 11 tipos de errores distintos y, por lo tanto, el circuito de detección de errores se repite 11 veces para cada fichero de datos. Al mismo tiempo, en este proceso, se genera una salida gráfica a nivel de marcadores con un fichero global de errores. Para ello se carga en el entorno SIG su componente geométrica junto con un estilo predefinido para poder diferenciar el tipo de error.

En la Figura 9 se ejemplifica el resultado de todo ello, mostrando los marcadores y líneas generados para poder localizar los errores. Esta es la información gráfica, que posteriormente se exportará a DXF, se vinculará al entorno CAD y se usará como soporte para la corrección de error.

A modo de resumen, los ficheros resultantes del proceso son:

- Fichero de texto con un resumen global de errores. Contiene un informe de las hojas procesadas en el que se incluye la relación de tipos de errores encontrados en cada una y el computo de errores de cada tipo que presentan. 


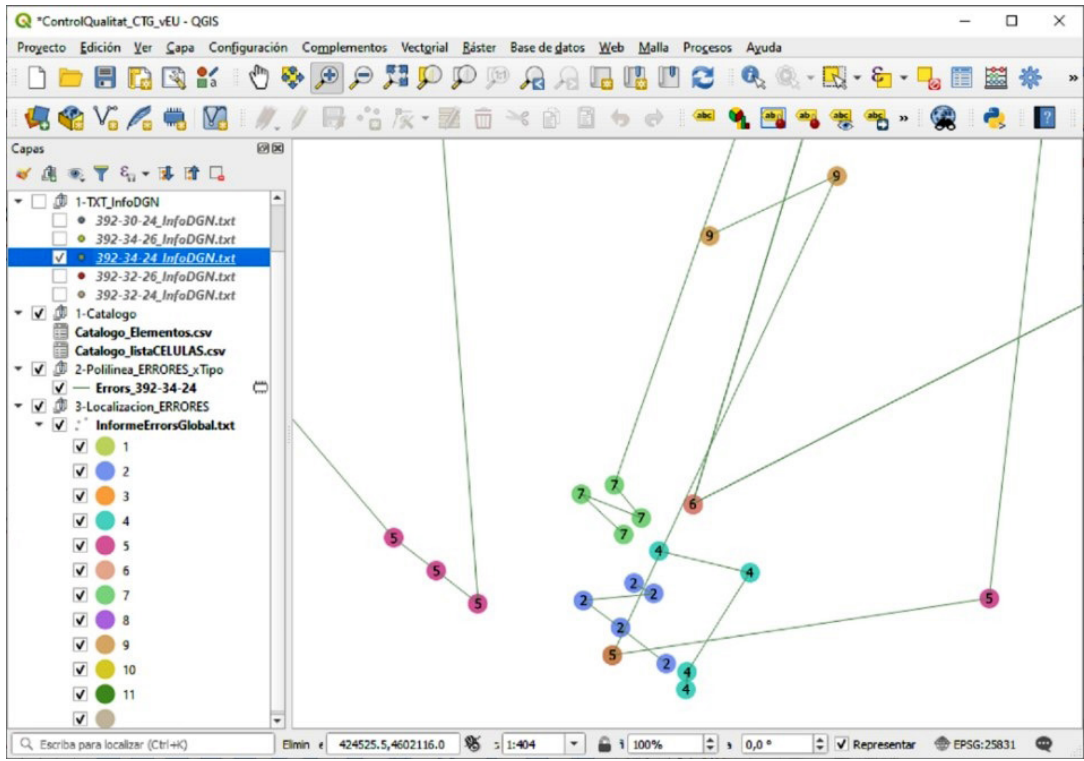

Figura 9. Ejemplo de resultado del proceso de detección de errores.

- Fichero de texto con un listado global de errores. Contiene información del identificador del elemento que presenta error, la hoja en la que se encuentra, el tipo de error detectado y otras características del elemento.

- Fichero DXF que incorpora la distribución de las marcas de error.

En este punto finaliza la fase del control de calidad y puede comenzar la fase de corrección de errores en el entorno CAD (fase 4).

\subsubsection{Corrección de errores (fase 4)}

Esta última fase se desarrolla en entorno CAD y engloba el conjunto de acciones necesarias para corregir los errores detectados en el control de calidad. En la Figura 10 se recoge la superposición de las marcas de error (exportadas como un fichero DXF) con las hojas cartográficas. Cada círculo representa un error detectado y el número que contiene indica el tipo de error que se ha encontrado en ese elemento.

Antes de definir cómo será la corrección de errores se hace necesario realizar una serie de consideraciones sobre cómo abordar la corrección de cada tipo de error.

De modo general, los errores de los tipos 1, 2 y 3 indican elementos que no se encuentran en el nivel (capa) que les corresponde y deberán ser reasignados manualmente, ya que la casuística puede ser muy variada.

Por otro lado, el error del tipo 4, que localiza elementos lineales de longitud cero, hace necesaria la eliminación de estos elementos de dibujo, ya que se 


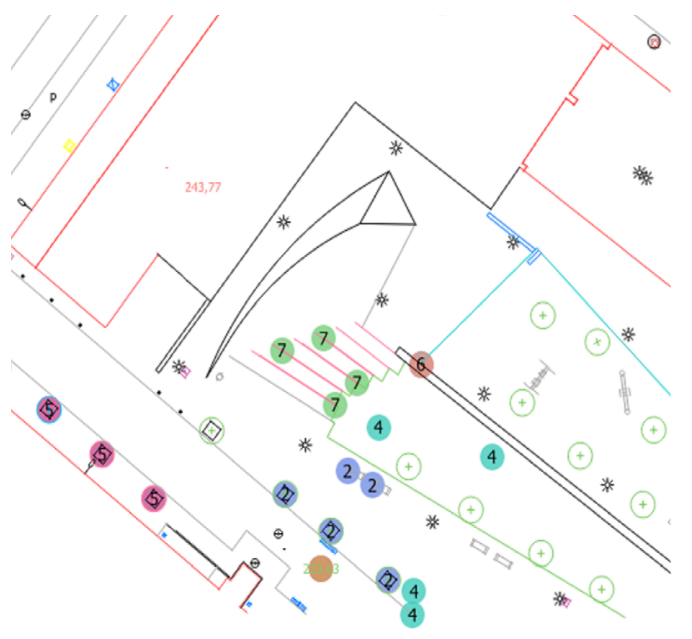
Leyenda de tipo de errores
1 Error 1-Nivel lineal con otros tipos de elementos
2 Error 2-Nivel puntual con otros tipos de elementos
Error 3-Nivel de Texto con otros tipos de elementos
Error 4-Elementos lineales de longitud cero
Error 5-Elementos con color diferente al asignado
Error 6-Elementos con tipo de línea diferente al asignado
Error 7-Elementos que con grosor diferente al asignado
Error 8-Elementos puntuales que no deberían estar orientados
Error 9-Niveles de T-cota_altimétrica_ * con formato inesperado (\#\#\#,\#\#)
Error 10-Nivel T-cota_corba_nivell con con formato inesperado (\#\#\#,\#)
11 Error 11-Clase no "primaria"

Figura 10. Ejemplo de superposición de las marcas de error en una hoja cartográfica (arriba) y leyenda asociada (abajo).

trata de elementos residuales definidos por un único punto y que, en realidad, no representan nada.

Los errores del tipo 5, 6 y 7 son errores de representación de los elementos, que deberían estar definidos en función del nivel que los contiene. Dado que el control de calidad está indicando que hay elementos en esa hoja que presentan por lo menos un error de este tipo, se resolverá forzando todos los elementos del fichero a que se representen según las propiedades concretas de los niveles.

Los errores del tipo 8 indican elementos puntuales de tipo células que están girados pero que no deberían estar orientados, por lo que en estos casos se deberá localizar el elemento y forzar la orientación con valor cero.

Los errores del tipo 9 y 10 son errores relacionados con el formato del texto. Se comprueban valores de cotas altimétricas y se valida que el separador decimal sea la coma y el número de posiciones decimales sea el esperado en función del nivel en el que se encuentran. 
Finalmente, el error del tipo 11 hace referencia a un valor de MicroStation, cuyo comportamiento esperado sería que todos los elementos pertenecieran a la clase "primaria". En caso de no cumplirse será necesario localizar el elemento y modificar este valor.

La heterogeneidad de los errores y de las soluciones para corregirlos hace difícil la automatización total del proceso. Por ello, la metodología propuesta se centra en utilizar herramientas que ayuden a localizarlos con precisión y rapidez. En este sentido se plantean dos avances. Por un lado, y como hemos descrito, la superposición de las marcas de error sobre las hojas cartográficas, que ayuda notablemente al operador a identificar fácilmente los elementos afectados por error. Por otro lado, la implementación de macros que permitan identificar un elemento en concreto o seleccionar un grupo de elementos a tratar y gestionar de modo masivo la corrección según la naturaleza del error.

\section{Caso de estudio y resultados}

La metodología y herramientas presentadas se aplicó al caso del municipio de Sabadell (web.sabadell.cat) en la provincia de Barcelona. La cartografía topográfica elaborada por el ayuntamiento de Sabadell es una cartografía a gran escala (1:500 y 1:1 000) que pretende dar respuesta a las necesidades diarias de la gestión municipal. Dicha cartografía se ha ido generando y actualizando parcialmente con vuelos fotogramétricos de diferentes zonas del municipio, así como mediante levantamientos topográficos realizados por el equipo de topografía del ayuntamiento de Sabadell. Todo el procedimiento de restitución, levantamiento y revisión está recogido en el Pliego de especificaciones técnicas de la cartografía topográfica 2D del Ayuntamiento de Sabadell a escalas 1:500 y 1:1 000.

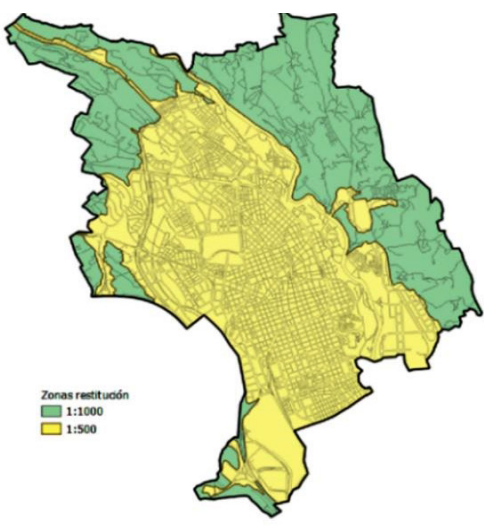

(a)

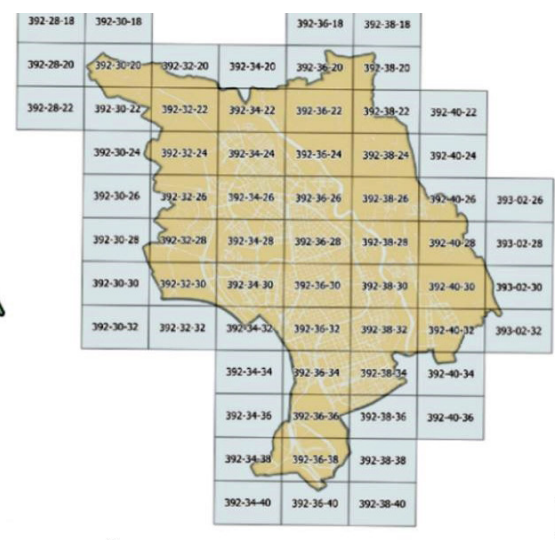

(b)

Figura 11. a) Cartografía municipal del ayuntamiento de Sabadell; b) Hojas de la serie cartográfica.

Fuente: Ayuntamiento de Sabadell. 
La cartografía de Sabadell se presenta cortada por hojas según el formato 1:2 000 ajustado al término municipal, conformando un total de 65 hojas (Figura 11).

\subsection{Ejemplo del procedimiento de detección de errores}

Como fase inicial se partirá del proyecto de QGIS de inicio que consta de tres capas de soporte cargadas: el límite municipal, la estructura de manzanas y la cuadrícula de la serie cartográfica. Asimismo, el proyecto ya tiene cargados los scripts que necesitamos para pasar el proceso de detección de errores:

\section{Script 1}

Este proceso genera una estructura en el árbol de QGIS para ordenar los datos que se van a procesar. Por un lado, el proceso importa los ficheros TXT con la información exportada de las hojas cartográficas. Y, por otro lado, el script importa las tablas estructuradas del catálogo de representación elementos.

Después de incorporar los datos de partida al proyecto de QGIS, el script enlaza los datos del catálogo con cada uno de los ficheros TXT de las hojas cartográficas mediante una unión de tablas virtual (usando los campos de unión que anunciamos en la Figura 6).

\section{Script 2}

El segundo script ejecuta el proceso de detección de errores sobre los ficheros cargados al proyecto en base a los tipos de errores definidos. En este punto el técnico puede decidir si quiere buscar errores en todos los ficheros o si prefiere lanzar el proceso sobre uno o varios de los ficheros cargados. En la Figura 12 se ejemplifica el proceso sobre seis ficheros de la cartografía municipal de Sabadell.

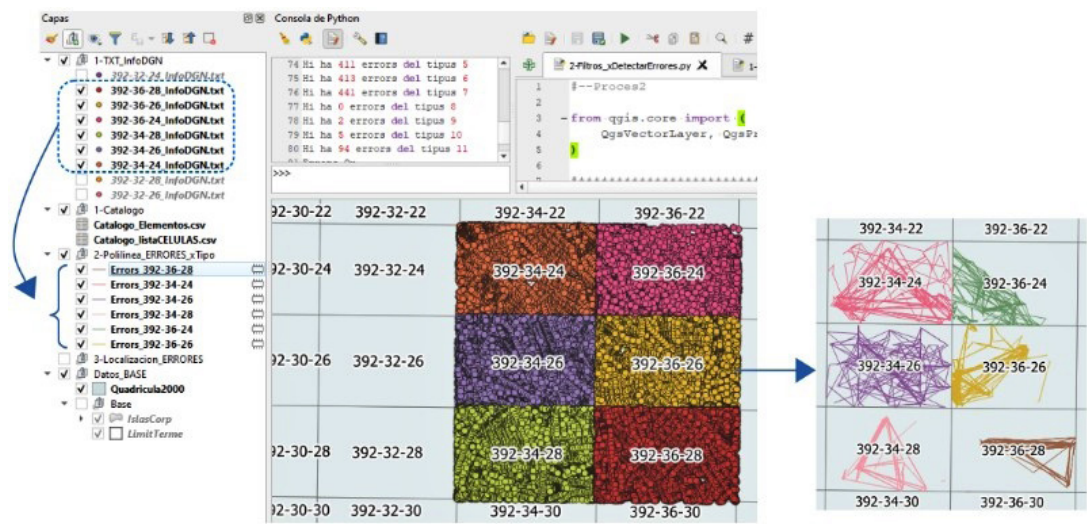

Figura 12. Resultado de la ejecución del segundo script de Python. 
La tabla de atributos de una de estas capas resultantes contiene un registro de entrada por cada tipo de error detectado. Cada uno de estos registros corresponde a un elemento lineal creado con las coordenadas de todos los elementos que presentan error agrupadas por tipos de errores (Figura 13).

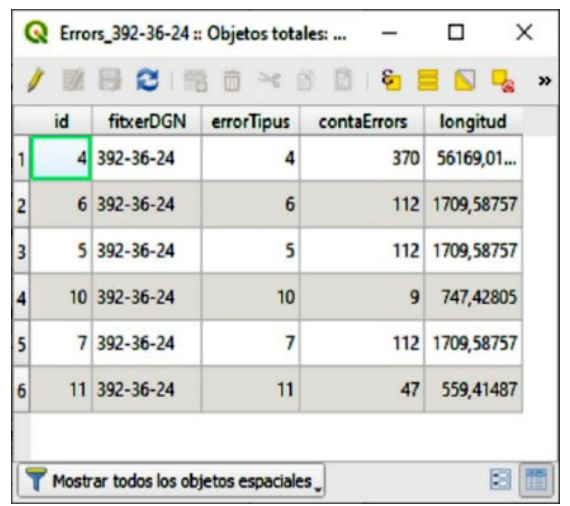

Figura 13. Tabla de atributos de la capa Errors_392-36-24.

Este script también genera listados de los errores que se han localizado.

\section{Script 3}

Antes de comenzar la corrección de errores, se crearán los marcadores con la localización de los errores para exportarlos como un DXF con el objeto de facilitar la corrección de las hojas en el entorno CAD. El resultado de ejecutar este script es una distribución de marcas circulares con un identificador numérico del tipo de error. En la Figura 14 se recogen distintas formas de visualizar las marcas de los errores detectados.

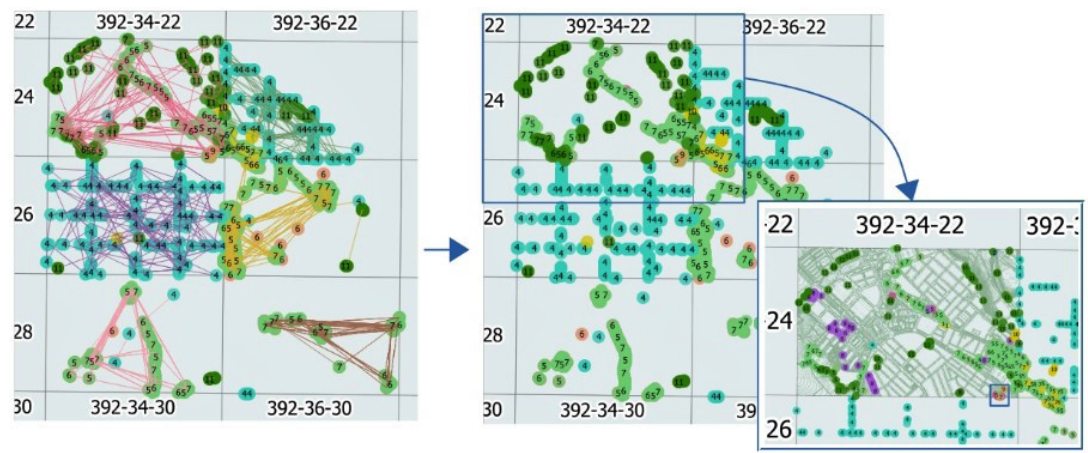

Figura 14. Visualización de las marcas de errores detectadas. 


\section{Exportación}

Tras la ejecución de los scripts, el siguiente paso consiste en exportar las marcas como un fichero DXF. Este proceso no está automatizado porque se realiza a partir de una única petición y, de este modo, el técnico puede decidir si quiere exportar todas las marcas o seleccionar las que quiere tratar en ese momento, pudiendo repetir el proceso de exportación tantas veces quiera y modificando la selección de marcas según su propio criterio. También se pueden crear las marcas para una única hoja.

\subsection{Ejemplo del procedimiento de corrección de errores}

A modo de ejemplo se describe la realización de correcciones sobre las hojas tratadas en el apartado anterior. Las correcciones se realizan directamente sobre los ficheros DGN de MicroStation siguiendo los siguientes pasos:

1. Apertura del fichero con el resumen global de errores Inspección del fichero resumen con el cómputo de errores, para identificar si hay errores que se puedan corregir de forma masiva.

2. Depuración de errores groseros en MicroStation

Por un lado, para el ejemplo planteado, es posible forzar la representación de los elementos para que sigan los atributos definidos por la capa que los contiene y corregir así los errores del tipo 5, 6 y 7 que en el presente ejemplo aparecen en cinco de las seis hojas procesadas. Con esta acción se eliminarán de forma masiva muchos de los errores detectados en las hojas. Por otro lado, podemos solventar el error tipo 4 (capas lineales con objetos de longitud cero) en las hojas en las que aparece. Se puede ejecutar una macro definida con VBA para eliminar este tipo de error y evitar localizar uno a uno dichos elementos residuales del dibujo.

3. Utilización de macros de limpieza de errores

En general, cuando el número de errores de un tipo sea alto, es muy recomendable disponer de herramientas que localicen los errores a partir de un listado y que se ejecute una acción para corregir el error de forma automática en el entorno CAD.

Para ello el técnico tendrá que preparar un listado con los identificadores de los elementos a tratar y ejecutar la macro correspondiente sobre las hojas que presenten ese error. El técnico podrá generar el listado desde el proyecto de QGIS filtrando los datos del error requerido en la capa de marcadores, y exportar un listado específico para cada hoja con la tipología de error y los identificadores pertinentes.

4. Creación de los nuevos ficheros DGN corregidos y re-ejecución del proceso de detección de errores en el QGIS

Después de las correcciones de errores realizadas en este ejemplo, se volverán a exportar los datos de estas hojas y a ejecutar nuevamente el proceso de detección de errores en QGIS. El hecho de trabajar con un fichero ya depurado permitirá al técnico municipal centrarse en los errores concretos que aparezcan en las hojas. 
En la Figura 15 se ejemplifica el resultado de los errores detectados al ejecutar de nuevo el proceso de detección de errores.

5. Corrección de errores concretos en el entorno CAD (MicroStation)

En esta fase se corregirían los errores que no pueden ser automatizados mediante una macro. Para ellos se localizaría los errores del tipo 1 y 8 , y se corregirán de forma manual y puntual (Figura 16).

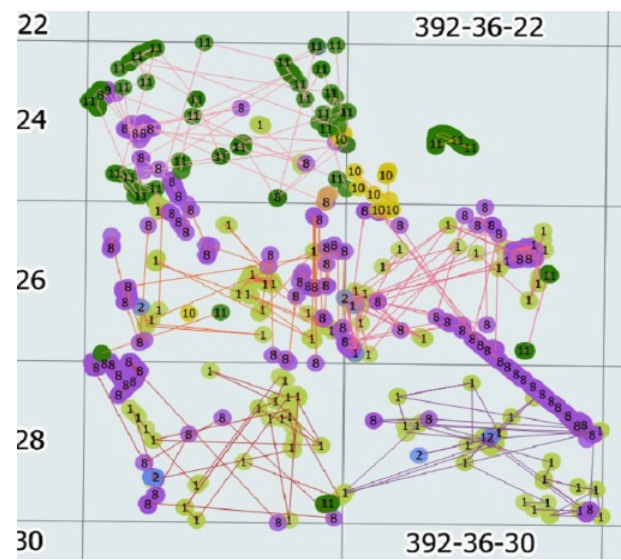

Figura 15. Resultado de la detección de errores, después de corregir los tipos 4 a 7.

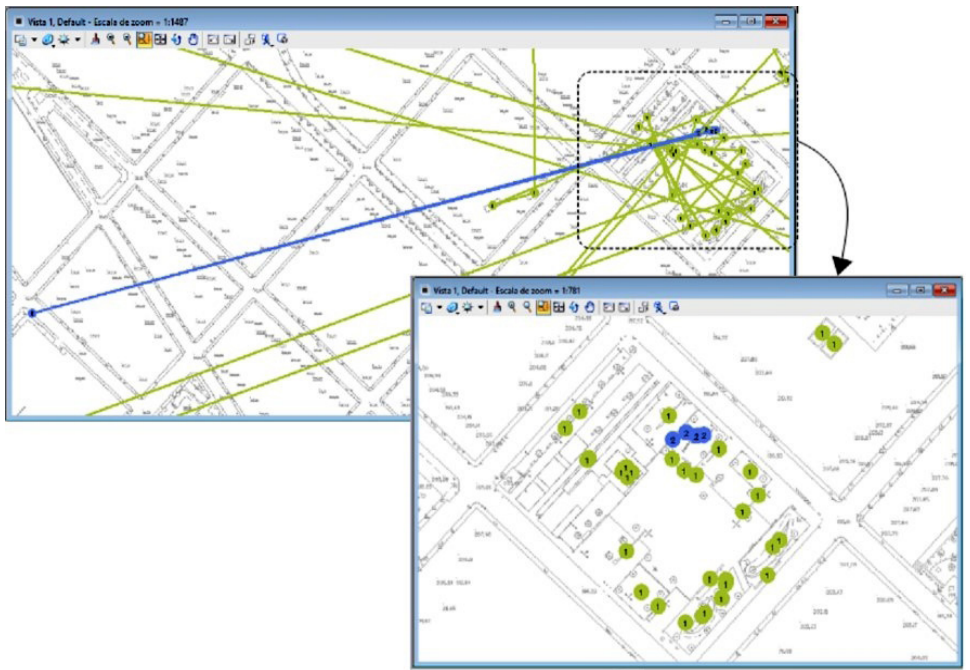

Figura 16. Localización de los errores del tipo 2 en la hoja 392-36-28. 
6. Vuelta al paso 4 y repetición del proceso

En este punto, se volvería a realizar la exportación de datos de los DGN revisados para comprobar que el proceso de detección de errores ya no encuentra ningún tipo de error $y$, por lo tanto, las hojas han quedado revisadas y corregidas.

\section{Conclusiones}

El objetivo principal del artículo ha sido la automatización del proceso de control de calidad de una serie cartográfica al objeto de optimizar tiempos, recursos y evitar tareas repetitivas asociadas a la revisión manual. Para ello se ha establecido una metodología que permite definir las condiciones que deberían cumplir los elementos representados en la cartografía y localizar aquellos que no siguen la norma. El procedimiento de control de calidad definido se puede ejecutar de forma global sobre todas las hojas de la serie cartográfica o sobre aquellas hojas que se han modificado para ajustarlas a la realidad del territorio y detectar si se han producido errores durante el proceso de actualización.

Se ha tratado de que los técnicos intervengan lo mínimo en la detección de errores y se liberen de la preparación de los datos de control, que quedan establecidos en la fase inicial del proyecto. En este sentido, el técnico únicamente debe indicar las hojas sobre las que quiere ejecutar el proceso y realizar la corrección a partir de los ficheros resultantes del proceso de detección de errores. Una vez que los técnicos han corregido las hojas según las marcas de errores, se puede volver a pasar el proceso de control de calidad sobre las hojas revisadas para garantizar que se han corregido todos los errores detectados inicialmente. En todo caso, debería repetirse este proceso tantas veces como fuera necesario hasta que los ficheros resultantes del proceso indiquen que no se detecta ningún tipo de error y que las hojas han quedado completamente limpias.

Al aplicar este proceso de control, se reduce el tiempo de ejecución de la revisión de errores de la serie cartográfica ya que hay varios procesos que se pueden ejecutar por lotes. En términos generales, se calcula que los tiempos de detección de errores se han reducido considerablemente, estimándose empíricamente en un $80 \%$, garantizando que no se producen descuidos y que se revisa por igual todos los tipos de error definidos en toda la serie o hojas procesadas. Los técnicos ahorran tiempo ya que con el nuevo proceso se agrupan fases de trabajo y se evitan secuencias de trabajo encanadas y repetitivas eliminándose descuidos y errores.

Además, el fichero resumen de errores permite decidir la técnica de corrección a aplicar. Estas técnicas de corrección pueden llevarse a cabo bien corrigiendo los errores detectados mediante procesos de limpieza predefinidos, o bien de forma concreta cuando hay pocas apariciones de un tipo de error y el técnico decide realizar la corrección de forma manual apoyándose en la geolocalización de estos errores.

En el presente acercamiento se han definido once tipologías de error, pero es susceptible de ser fácilmente escalado. No obstante, con estas once categorías 
de error se ha demostrado la viabilidad de utilizar el entorno SIG para definir un proceso de control de calidad y de la metodología establecida.

A modo de perspectivas futuras, los ficheros de errores resultantes, se podrían utilizar para evaluar otras medidas de calidad. Por ejemplo, cruzando la información obtenida con los editores de cada hoja cartográfica, se podría detectar si se producen errores sistemáticos asociados a un editor concreto o si es un error generalizado y se repite por todos los editores de cartografía, cosa que podría alertar de algún problema con la plantilla de trabajo u otras casuísticas. Es decir, analizando los ficheros de errores se podrían identificar componentes que afectan a la calidad cartográfica y aplicar medidas de corrección o definir actuaciones o procedimientos de trabajo para reducir la generación de errores y mejorar la calidad del producto final.

También se podría utilizar el SIG para identificar otras medidas de calidad. Por ejemplo, se podría obtener un listado de los distintos textos que se encuentran en el fichero. No solo para garantizar que el formato de los textos es el esperado en el caso de elementos genéricos que se utilizan para etiquetar estaciones transformadoras, rampas, etc., sino para conocer las toponimias específicas que hay en el dibujo y validar si su localización es correcta, etc.

Por todo lo expuesto se concluye que la migración del control de calidad al SIG para agilizar el proceso de detección y corrección de errores en series cartográficas es muy conveniente. La versatilidad, flexibilidad y escalabilidad de la metodología planteada, así como los futuros desarrollos apuntados, que pueden complementar lo expuesto en este artículo, le atribuyen un alto potencial de mejora para la gestión cartográfica de las corporaciones municipales.

\section{Agradecimientos}

Al Ayuntamiento de Sabadell por facilitar el acceso a las series cartográficas y a sus especificaciones técnicas, en especial a la sección de Cartografía del Servicio de Información de Base.

\section{Bibliografía}

Ariza, F. J., \& Rodríguez, A. F. (2018). Calidad en datos geográficos, geoservicios y productores de datos: análisis crítico. Revista Cartográfica, 97, 105-143.

https://doi.org/10.35424/rcarto.i97.178

Ariza-López, F. J. (Ed.) (2013). Fundamentos de evaluación de la calidad de la información geográfica. Jaén, España. Servicio de Publicaciones de la Universidad de Jaén.

Bentley Systems (2020). Microstation V8i.

Recuperado de: https://www.bentley.com/es/products/brands/microstation

Comissió de Coordinació Cartogràfica de Catalunya (2016). Plec d'especificacions tècniques per a l'elaboració de cartografia topogràfica 2D i 3D a escala 1:500 (CT-5C) v2.0. Recuperado de:

https://www.icgc.cat/index.php/content/download/58677/408654/version/15/ file/topografia-500-v2r0-esp-02ca-20161101.pdf

Diario Oficial de la Generalitat de Catalunya. (2010). Decreto 62/2010, de 18 de mayo, por el que se aprueba el Plan Cartográfico de Cataluña. Recuperado de: 
https://dogc.gencat.cat/es/pdogc_canals_interns/pdogc_resultats_ fitxa/?action=fitxa\&documentld=542412\&language=es_ES

Gallego-Priego, M. (2017). Aplicación de los fundamentos de las infraestructuras de datos espaciales en la construcción de sistemas de información geográfica corporativos. Tesis doctoral, Universidad de Salamanca, Ávila.

QGIS Development Team (2020). QGIS Geographic Information System. Open Source Geospatial Foundation Project. Recuperado de http://qgis.osgeo.org 